\title{
Wind Turbine System : An Industrial Case Study in Formal Modeling and Verification
}

\author{
Jagadish Suryadevara ${ }^{1}$, Gaetana Sapienza ${ }^{2}$, \\ Cristina Seceleanu $^{1}$, Tiberiu Seceleanu ${ }^{2}$, Stein-Erik Ellevseth ${ }^{2}$, and Paul Pettersson ${ }^{1}$ \\ 1 Mälardalen Real-Time Research Centre, Mälardalen University, Västerås, Sweden. \\ \{jagadish.suryadevara, cristina.seceleanu, paul.pettersson\}@mdh.se \\ 2 ABB Corporate Research. \\ \{gaetana.sapienza, tiberiu.seceleanu\}ese.abb.com, \\ stein-erik.ellevseth@no.abb.com
}

\begin{abstract}
In the development of embedded systems, the formal analysis of system artifacts, such as structural and behavioral models, helps the system engineers to understand the overall functional and timing behavior of the system. In this case study paper, we present our experience in applying formal verification and validation $(\mathrm{V} \& \mathrm{~V})$ techniques, we had earlier proposed, for an industrial wind turbine system (WTS). We demonstrate the complementary benefits of formal verification in the context of existing $\mathrm{V} \& \mathrm{~V}$ practices largely based on simulation and testing. We also discuss some modeling trade-offs and challenges we have identified with the case-study, which are worth being emphasized. One issue is related, for instance, to the expressiveness of the system artifacts, in view of the known limitations of rigorous verification, e.g. model-checking, of industrial systems.
\end{abstract}

Keywords: Industrial Case-Study, Wind Turbine System, MARTE/CCSL, EAST-ADL, Verification, Model Checking, UPPAAL

\section{Introduction}

The increasing complexity and criticality of real-time embedded systems (RTES), in domains such as industrial automation, automotive and avionics, stresses the need for applying systematic design phases, combined with rigorous verification and validation $(\mathrm{V} \& \mathrm{~V})$ techniques, during system development [3]. A well-defined design process with necessary tool support leads to ensuring system predictability, w.r.t intended functional and timing behavior. Nevertheless, meeting such a clear objective has several challenges. One of pre-requisites is well-defined system artifacts representing system structure as well as behavior with reactive, continuous, discrete, and real-time features, or a combination thereof, at suitable levels-of-abstraction. For complex industrial systems, the above design by-products, while necessary, may lead to additional issues such as ensuring traceability, analyzability as well as reusability of the system artifacts. In this context, model-based development approaches, which enable continuous V\&V throughout the development process, have become a feasible solution to tackle some of the challenges. However, formal verification techniques such as model checking, while 
useful for the exhaustive analysis of system behavior, are challenging to apply for complex system models. A related issue is choosing a suitable level of granularity and expressiveness for system artifacts, given the well-known limitations of model-checking, such as the state-space explosion problem. In this paper, we address some of these challenges in the context of applying modeling and formal verification techniques using a wind turbine system case-study, a complex industrial RTES.

The Unified Modeling Language (UML) provides a modeling profile called MARTE (Modeling and Analysis of Real-Time and Embedded systems) [7] to support the performance and schedulability analysis of system models. MARTE also includes CCSL - a time model and a clock constraint specification language [1] for specifying logical and chronometric constraints for system models. On the other hand, EAST-ADL [2], an emerging standard for automotive systems, provides an integrated model-based development for RTES, through well-defined phases, as well as support for traceability. Recently, EAST-ADL has been integrated with model-checking support for componentbased designs, e.g. the ViTAL tool [4] based on the timed automata technology for verification $[5,11,10]$.

In this paper, we target the verification of functionality and timing behavior of a wind turbine system developed in the context of the iFEST (industrial Framework for Embedded Systems Tools), an ARTEMISIA project. In Section 2.2, we overview a simplified version of the wind turbine system (WTS), and describe its functionality and timing behavior. Rest of the paper is organized as follows: In Section 3, we briefly recall CCSL and timed automata. In Section 4, we describe a modeling methodology for the WTS to enable verification using model checking. The analysis results of simulating, as well as model checking the WTS model are presented in Section 5. In Section 6, we discuss our experience with the case study with respect to the challenges and limitations in applying formal techniques to complex industrial systems. We conclude the paper in Section 7.

\section{Windturbine System (WTS) : An overview}

Wind energy sources are fast-growing and in line with the technological advancement. Modern wind turbine systems require sophisticated and effective control functionalities in order to fulfill performance, safety, and maintainability requirements. The main purpose of a wind turbine system is to convert the rotational mechanical energy of the rotor blades (i.e. mechanical components of a wind turbine) caused by the wind into electrical energy to be redistributed via a power grid. Given the system's complexity, the iFEST (industrial Framework for Embedded Systems Tools) project ${ }^{3}$ aims at providing a model-based approach for system development, to ensure the system predictability w.r.t the specified functional and timing behavior.

\subsection{Development Process and Environment}

In the iFEST project, we have carried out the system development by adopting the Vmodel based software development approach, as follows:

\footnotetext{
${ }^{3}$ http://www.artemis-ifest.eu/
} 
During Requirement and Analysis phase, we have documented the WTS requirements, both functional and extra-functional including timing behavior. For the Design phase, we have combined component- and model-based approaches, keeping in view the overall system analyzability and reusability requirements. During the Implementation phase, we have applied automatic code generation technologies.Subsequently, the implemented system, a combined FPGA and CPU solution, has been deployed on a heterogenous hardware platform (XilinX ZynQ 7000 product family). For the Verification and Validation $(\mathrm{V} \& \mathrm{~V})$, we have used model-based techniques as follows: (i) simulation of the WTS functionality using Simulink and related toolboxes, and (ii) automatic model-based test-case generation with MaTeLo tool. However, the above techniques are not sufficient to ensure system predictability w.r.t to all possible system executions, hence formal verification is desirable to complement the current analysis methods. To address the above open issue, in this paper, we present a verification technique towards enhanced system validation. And, our contributions are as below:

- As enhanced system validation, we apply verification technique to establish system properties, (partially) based on simulation results of Simulink-based system models.

- We are able to verify safety requirements that involve timing behavior (e.g. "the wind turbine moves to Park mode, within 30s of detecting that the wind speed has crossed the upper limit of $20 \mathrm{~m} / \mathrm{sec"}$ ).

\subsection{The Wind Turbine System Model}

The wind turbine system is modeled as a closed-loop control system, as shown in Figure 1. The key components are the Plant and the Controller subsystems. The Controller dynamically regulates the rotor blades of the wind turbine w.r.t the specified wind profile, to maximize the generation of electrical energy and also to avoid damage to the plant in case of turbulent wind scenarios. It automatically changes the Controller Output signal to regulate the plant, based on the wind and the plant's actual conditions, which are received by the Controller via the Sensor Input signals. The Wind Profile and the Resistive Load are used to simulate and test the behavior of the plant and the controller, under specific wind and resistive load conditions. Further details of the plant and controller subsystems are described below.

2.2.1 Plant model. As shown in Figure 2 (in Section 4), it consists of three main components; Servo, Rotor, and Generator. The pitch of the turbine, determined by the Controller (described below), is actuated by the Servo. The Rotor produces the required torque to maximize the angular speed of the Generator (which produces the final voltage), based on the pitch value as well as the current wind speed (we assume a fixed resistive load). The Rotor optimizes the produced torque value based on the current angular speed of the Generator.

2.2.2 Controller model. As shown in Figure 3 (in Section 4), it consists of four main components: the Filter, the Main Controller, the Pitch Controller, and the Park 


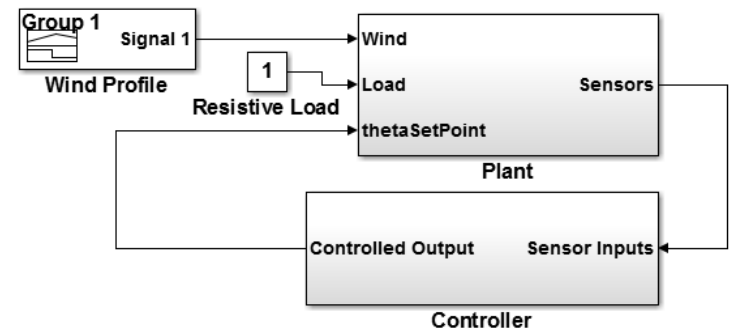

Fig. 1. Wind Turbine System Model

and Brake Controller. The Filter Subsystem is responsible for transducing, filtering and scaling the wind signal and plant signal (for instance the rotational speed of the turbine), which are used by the Main Controller and the Pitch Controller. Based on the inputs received through the Filter, the Main Controller directs the overall control. It oversees the performance and operations of the turbine in order to maximize the energy production and prevent any damage to the plant. Based on the wind and plant state, the controller determines the operational mode (i.e. park, start-up, generating, or brake) of the turbine. The Pitch Control calculates the proper pitch i.e. angle to steer the rotor blades when the turbine starts up or generates power. The Pitch and Brake controller determines if the turbine needs to brake or park, to ensure the safety of the wind turbine, for instance, during wind turbulances.

\section{Preliminaries}

In this section, we present an overview of the preliminaries needed for modeling of the wind turbine system. We have used EAST-ADL modeling framework for structural modeling of the WTS. The timed causality behavior of the system is specified using CCSL. To provide the verification using the UPPAAL, a model checking tool, we have developed the timed automata based semantic models of the system, based on the corresponding EAST-ADL models and the CCSL specifications.

\subsection{EAST-ADL}

The modeling process in EAST-ADL framework, developed in the context of the EASTEEA project, is structured into different abstraction levels such as feature level, analysis level, design level etc. At both analysis and design levels, the system is described by a FunctionalArchitecture that consists of a number of inter-connected FunctionPrototypes (instantiation of FunctionType components). FunctionProtoype components are either event- or time-triggered. The execution semantics of the EAST-ADL components is as follows; components interact through single buffer, rewritable, non-consumable ports, and execute in read-execute-write phases in run-to-completion fashion. The detailed timing behavior as well as timing constraints for an EAST-ADL model can be specified using TADL2, the Timing Augmented Description Language (ver 2), currently being integrated with EAST-ADL framework [8]. In related works, we have proposed verification techniques for TADL2-based EAST-ADL models $[5,11,10]$. 


\subsection{CCSL}

CCSL is used to specify the constraints imposed on the logical clocks (activation conditions) of a model. A CCSL clock is defined as a sequence of instants (event occurrences). CCSL constraints are of three kinds: (i) Synchronous constraints rely on the notion of coincidence. For example, the constraint "a coincidesWith b", denoted by $\mathrm{a}=\mathrm{b}$, specifies that each instant of a coincides with the corresponding instant of b. Another example of a synchronous constraint is "a isPeriodicon b period $n$ ", which specifies the subclock a whose 'ticks' correspond to every $n^{\text {th }}$ 'tick' of b. (ii) Asynchronous constraints are based on instant precedence; the constraint "a isfasterThan b" (denoted by a $\preccurlyeq$ b) specifies that clock a is (non-strictly) faster than clock b. (iii) Mixed constraints combine coincidence and precedence; the constraint " $\mathrm{c}=\mathrm{a}$ delayedFor $\mathrm{n}$ on $\mathrm{b}$ " specifies that $\mathrm{c}$ 'ticks' synchronously with the $n^{\text {th }}$ 'tick' of b following a 'tick'.

\subsection{Timed Automata}

A timed automaton is a tuple $\left\langle L, l_{0}, C, A, E, I\right\rangle$, where $\mathrm{L}$ is a set of locations, $l_{0} \in L$ is the initial location, $\mathrm{C}$ is the set of clocks, $\mathrm{A}$ is the set of actions, synchronization actions and the internal $\tau$-action, $E \subseteq L \times A \times B(C) \times 2^{C} \times L$ is a set of edges between locations with an action, a guard, a set of clocks to be reset, and $I: L \rightarrow B(C)$ assigns clock invariants to locations. A location can be marked urgent (u) or committed (c) to indicate that the time is not allowed to progress in the specified location(s), the latter being a stricter form indicating further that the next transition can only be taken from the corresponding location(s) only. Also, synchronization between two automata is modeled via channels (e.g., $\mathbf{x}$ ! and $\mathbf{x}$ ?) with rendezvous or broadcast semantics.

The UPPAAL model-checker extends the timed automata language with a number of features such as global and local (bounded) integer variables, arithmetic operations, arrays, and a C-like programming language. The tool consists of three parts: a graphical editor for modeling timed automata, a simulator for trace generation, and a verifier for the verification of a system modeled as a network of timed automata. A subset of CTL (computation tree logic) is used as the input language for the verifier. For further details, we refer to UPPAAL tutorial [6].

\section{WTS: Formal Specification and Modeling}

In this section, we present a formal specification and modeling approach for WTS, an aposteriori modeling technique, that is, the specification and modeling artifacts are based on existing design artifacts such as Simulink models, requirements documents etc. However, we apply an abstraction strategy to obtain the corresponding real-time semantic models that represent the system functionality as well as the timing behavior. Further, the strategy attempts to preserve the models' tractability to make the exhaustive verification feasible. The overall modeling strategy, based on design principles such as separation-of-concerns and correctness-by-construction, captures the underlying model-of-computation and the execution behavior of the WTS. Below, we outline some generic views/assumptions on which we base our formal modeling: 
- Plant models and Instantaneous executions. A Plant model represents physical devices such as sensors and actuators with the corresponding model-ofcomputation based on reactivity and instantaneity.

- Controller models and Timed executions. Controllers contain software components based on timed model-of-computation, with explicit timing aspects, such as delay, execution time, end-to-end deadline etc, to be formally specified and modeled.

- Time and event triggering. The activation or triggering of RTES components is generally based on specific time or event ${ }^{4}$ occurrences. Plant components are eventtriggered (i.e. in response to occurrence of input data), whereas controller components are time- or event-triggered, this primarily being a design-choice.

- Run-to-completion. RTES components execute in run-to-completion steps, that is, in terms of read-execute-write cycles.

- Data and value semantics. Due to the associated models-of-computation, as described above, a data entity at a given 'instant', in the Plant or Controller, may correspond to two distinct value instants.

- Real-time features. The structural and behavioral models of RTES often fail to model real-time features, such as urgency, priority, and synchrony (explained later) w.r.t to the underlying execution model.

- Environment modeling. An environment is external to the system, representing physical parameters such as temperature, pressure, wind speed etc. To support formal verification, a modeling strategy based on non-determinism as well as the properties to be verified, is needed.

To obtain an expressive and verifiable semantic model of the WTS, we employ a component-based modeling approach, based on real-time formalisms such as CCSL and timed automata.The overall modeling approach is as follows:

- Data and event representations are made based on the structural models.

- The timed causality behavior of the system components, w.r.t the associated modelof-computation, is formally specified using CCSL constraints.

- The functional behavior of the components is modeled using an abstract finite-statemachine notation, and transformed into timed automata.

- The CCSL constraints are transformed into timed automata, and composed using the notion of synchronization product (described later).

Finally, a real-time semantic model of the overall system is obtained as a network (i.e., a parallel composition) of timed automata described above.

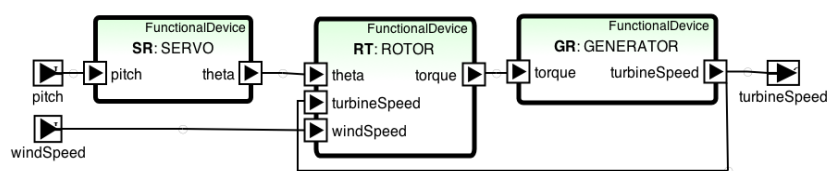

Fig. 2. Structural modeling: a plant model for the WTS.

\footnotetext{
${ }^{4}$ While time is also an 'event', we differentiate this in this paper explicitly.
} 
In Figure 2, we present the structural model of plant and controller for the WTS (based on the corresponding Simulink models), using the EAST-ADL modeling framework (in MetaEdit $+^{5}$ ). The main components of the plant, that is SERVO, ROTOR, and GENERATOR are modeled as FunctionalDevice prototypes in EAST-ADL. In Figure 3, we present the structural model of the Controller. It models the three sub-controllers MainControl, PitchRegulation, and ParkBrake, modeled as Analysisfunctiontypes. For further details of the functionality of these components, we refer to Section 2.2. We demonstrate the overall modeling approach for WTS, using the ROTOR and the MainControl components, below. We will also discuss some related modeling issues.

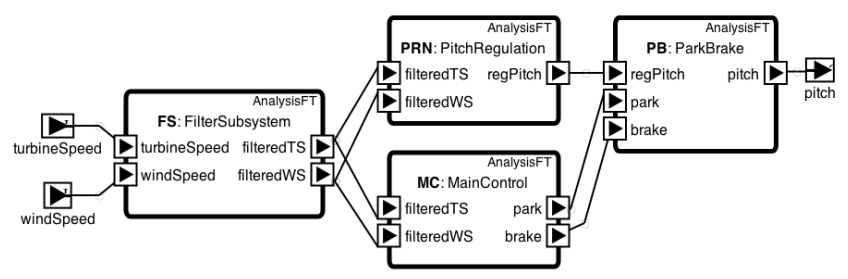

Fig. 3. Structural modeling: a controller model of the WTS.

\subsection{Data and Events}

As shown in Figure 2, the ROTOR prototype, denoted by RT, receives input pitch (theta), turbine speed (omega), and wind speed (ws) and produces the corresponding torque value as the output. Hence, we define the local variables thet $a_{l}$, omega $a_{l}$, and $w s_{l}$ and the corresponding global variables $w s_{g}$, omega $a_{g}$, thet $a_{g}$. The local variables are updated at the activation of the RT using the corresponding global values. This is consistent with the data semantics discussed previously.

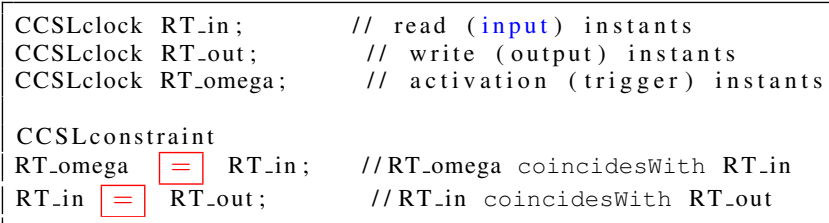

Listing 1.1. CCSL specification of ROTOR component.

\subsection{Specification of timed causality behavior}

The timed causality behavior of real-time components, w.r.t the corresponding model-of-computation, can be specified precisely using CCSL logical clocks and CCSL constraints. We use CCSL (logical) clocks to represent events corresponding to 'read', 'execute', and 'write' instants of a component. In Listing 1.1 and Listing 1.2 , we present the CCSL specification of ROTOR (RT) and MainControl (MC)

\footnotetext{
${ }^{5}$ www.metacase.com
} 
Table 1. Timing attributes of Controller components.

\begin{tabular}{|l|c|c|c|}
\hline Component & $\begin{array}{c}\text { Period } \\
(\mathrm{ms})\end{array}$ & $\begin{array}{c}\text { Min } \\
\text { Execution Time } \\
(\mathrm{ms})\end{array}$ & $\begin{array}{c}\text { Max } \\
\text { Execution Time } \\
\text { (ms) }\end{array}$ \\
\hline MainControl & 100 & 10 & 15 \\
PitchRegulation & 50 & 35 & 45 \\
ParkBrake & 50 & 15 & 20 \\
Filter & - & 20 & 25 \\
\hline
\end{tabular}

prototypes, respectively. The constraints specify the timed causality behavior of the components w.r.t to the corresponding model-of-computation. For instance, the CCSL constraints for RT specify the reactivity and instantaneity behavior of RT execution within the Plant model. On the other hand, the CCSL constraints for MC specify the time-triggered behavior of the controller execution. The timing attributes of the controller components are given in Table 1. The CCSL specifications provide a basis for constructing real-time semantic models e.g. timed automata based models, as well as observers to establish the system properties, as presented later in this section.

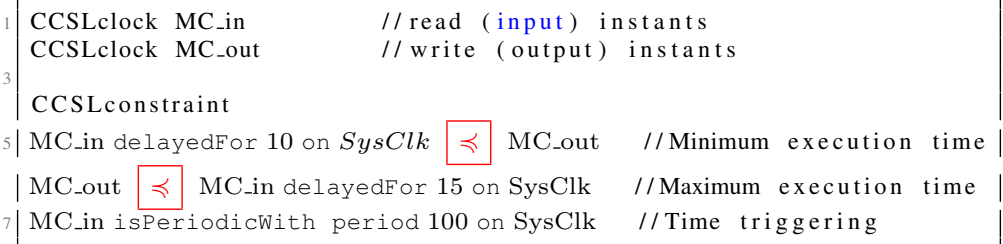

Listing 1.2. CCSL specification of MainControl component.

\subsection{Modeling functional behavior of real-time components}

In Figure 4, we present the behavior modeling for the MainControl protoype (based on the corresponding Simulink model). The behavior is specified using a finite-statemachine (FSM) notation. It represents the overall system behavior (stateful) in terms of control states PARK, START, BRAKE, and GENERATE. The states represent the operational modes of the WTS, based on the wind speed and the turbine speed; the mode transitions corresponding to mode-change behavior are triggered by boolean conditions (guards) $g 1, g 2, .$. etc. Further, we simply annotate the behavior model to denote the execution semantics such as run-to-completion (R-T-C) and history (denoted by the control node $\mathrm{H}$ ). The functionality of other components in the WTS are stateless computations, that is partial functions between input and corresponding output values, for instance as represented by the writeTorque() function of the ROTOR.

\subsection{Formal modeling of Plant components}

In this subsection, we present formal modeling approach, based on CCSL, for the plant components of the WTS. We had earlier proposed, in a previous work [10], 


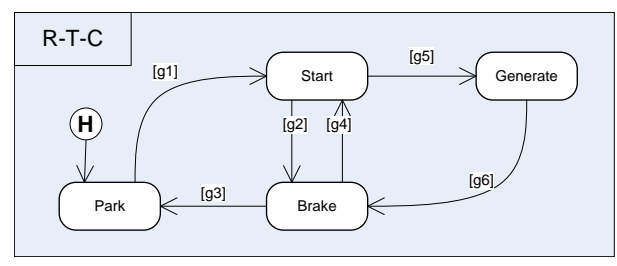

Fig. 4. Functional behavior of the MainControl component.

transformation of CCSL constraints into timed automata. The transformations can be used to derive timed automata based models that represent the timed causality behavior of the system. For instance, in Figure 5.(a) and 5.(b), we present the timed automata semantics of CCSL constraints that specify the timed causality behavior of ROTOR executions (see Listing 1.1), using events $\mathrm{RT}_{-}$in and RT_out representing component activation and termination respectively. Note that an event e.g. RT_in is modeled using synchronization channels i.e. send/receive signals RT_in! and RT_out!. Also note that the synchronous occurrence of event signals, e.g. GR_out? and RT_in! in Figure 5.(a), is specified using committed locations. A committed location indicates that the corresponding enabled transitions from the location are 'forced' before time can progress. This facilitates precise modeling of overall timing behavior of the system.

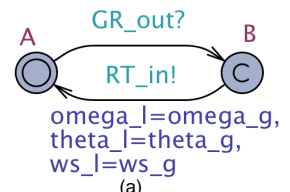

(a)

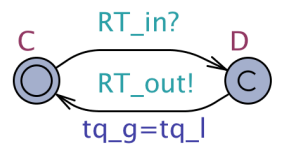

(b)

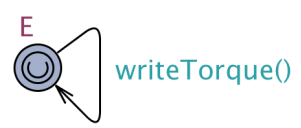

(c)

Fig. 5. Timed automata modeling: (a) RT_omega $=R T_{\text {_in }}$ (b) RT_in $=$ RT_out Computation RT.

The above automata can be composed, using the notion of synchronization product (based on common labels or synchronization signals), as shown in Figure 6.(a). For instance, locations B and $\mathrm{C}$ in the automata in Figure 5.(a) and Figure 5.(b) respectively, are mapped to the location $\mathrm{BC}$ in Figure 6.(a), due to the synchronization of signals RT_in! and RT_in?.
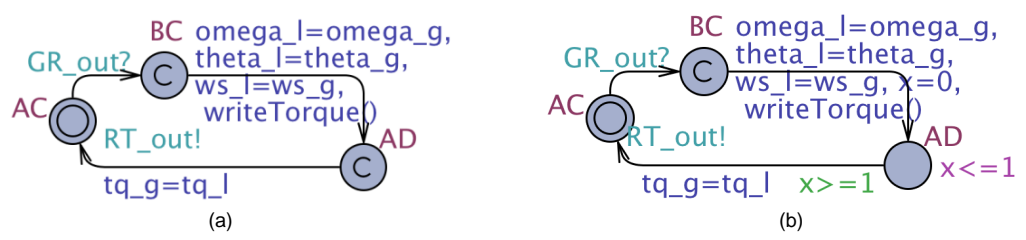

Fig. 6. Timed automata model for (event-triggered) ROTOR.

It can be noted that the composed location 'BD' is not possible in the synchronized product automaton, as the location is non-reachable due to the synchronization at $\mathrm{B}$ and $\mathrm{C}$, leading to location $\mathrm{AD}$ (i.e. location $\mathrm{A}$, and $\mathrm{D}$ simultaneously in resp. automata) in the synchronized product, instead. Further, as shown in Figure 6.(a), we 
associate the transitions corresponding to component activation, with data updates and the corresponding computation; the $\mathrm{RT}_{-}$in event denotes input as well as execution of the corresponding functionality, during a transition from location $\mathrm{BC}$ to location AD. However, to make the overall automata model of the WTS system tractable (time-wise), and hence formally verifiable, we need to relax the notion of instantaneity for the automata models of the Plant components. This can be done by introducing a minimum time delay for each component, if not specified already. This is done by assigning a timing invariant, the delay of one time unit, for instance at location AD in Fig.6.(b).

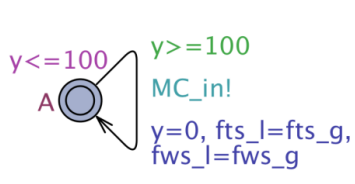

(a)

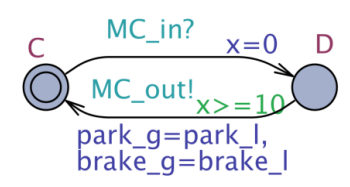

(b)

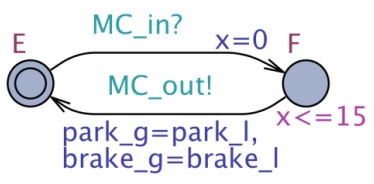

(c)

Fig. 7. Semantic modeling: (a) Periodic triggering $\quad$ (b) Min. exec. time $\quad$ (c) Max. exec. time

\subsection{Formal modeling of Controller components}

In this subsection, we describe the timed automata modeling of the Controller components for the WTS. In Figure. 7, we present the timed automata semantics of the CCSL constraints (Listing 1.2) that specify the time-triggered execution behavior of the MainControl (MC) prototype. We have composed these automata, as shown in Figure 8.(a), based on the notion of synchronization product (as described in the previous subsection). This consists of following steps; we have composed the automata in Figure 7.(b) and 7.(c), and then finally with the automaton in Figure 7.(a) (note the invariant $y \leq 100$ at every location in the product automaton).

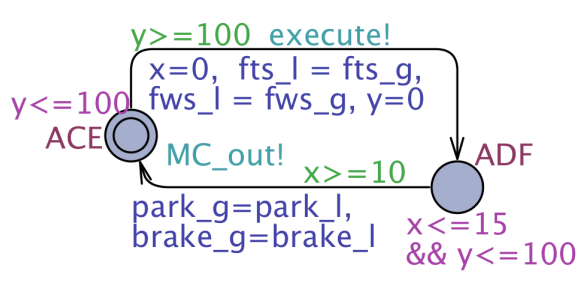

(a)

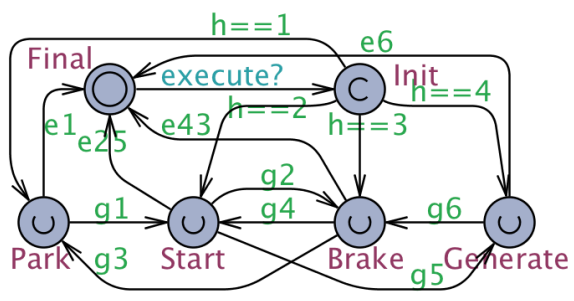

(b)

Fig. 8. Timed automata modeling of MainControl: (a) time-triggering (b) functional behavior.

As shown in Fig.8.(b), we have also transformed the behavior (functional) model of the MainControl (Fig.4) component into corresponding timed automaton, following the mapping techniques proposed previously [9]. We briefly outline the transformation as follows; we have mapped the control states to automaton locations. Further, using additional locations Init and Final and the history variable ' $h$ ', we have modeled the execution semantics, that is, run-to-completion, and preserving the history. For model 
readability, we have not shown the data updates for the transitions; also, the boolean guards of the form 'e $i j$ ' correspond to actual expression $(\neg \mathbf{g} i \& \& \neg \mathbf{g} j)$. It can be noted that all the locations of the transformed automaton are marked 'urgent' indicating the behavior model does not consume time, which has been separately modeled using the timed causality model discussed above. Finally, we 'connect' the transformed behavior model of the MainControl prototype, as described above, with the automata model of the corresponding timing behavior (Fig.8.(b)), using synchronization channel 'execute'.

\subsection{Modeling the WTS system}

Following the modeling strategy presented in the previous subsections, we can obtain the timed automata models for all the WTS components, and form a network (parallel composition) of these automata to obtain a timed automata based semantic model for the complete system. However, some issues exist as discussed below:

Modeling the Environment: The plant model described previously, models the components such as sensors and actuators constituting an environment model for the WTS controller. However, this is not sufficient to obtain a 'closed' model of the system that is necessary to enable exhaustive verification of the WTS model. For instance, modeling external parameters such as WindSpeed, while necessary, is not feasible using timed automata. In view of this, as well as the hybrid nature of the plant components e.g. ROTOR, GENERATOR etc, we choose to integrate the simulation data of the corresponding Simulink models, to construct the partial functions that represent the computations of the components.

Modeling 'observer' automata: The formal specification of complex properties of the system, while possible using CTL (the property specification formalism of UPPAAL), may not be directly verifiable. Instead, these can be intuitively modeled as observer automata, (parallel) composed with the main system model, and can be efficiently verified.

\section{WTS Analysis}

In this section, we present both simulation as well as the verification results for the WTS, and their correlation in verifying functional and safety-critical properties w.r.t the overall timing behavior of the system.

\subsection{Simulation}

The main purpose of simulating the WTS, using the MathWorks Simulink and StateFlow ${ }^{6}$, is to analyze the system behavior under normal operating conditions, and to validate the system (in particular the Controller) when the wind speed exceeds the 


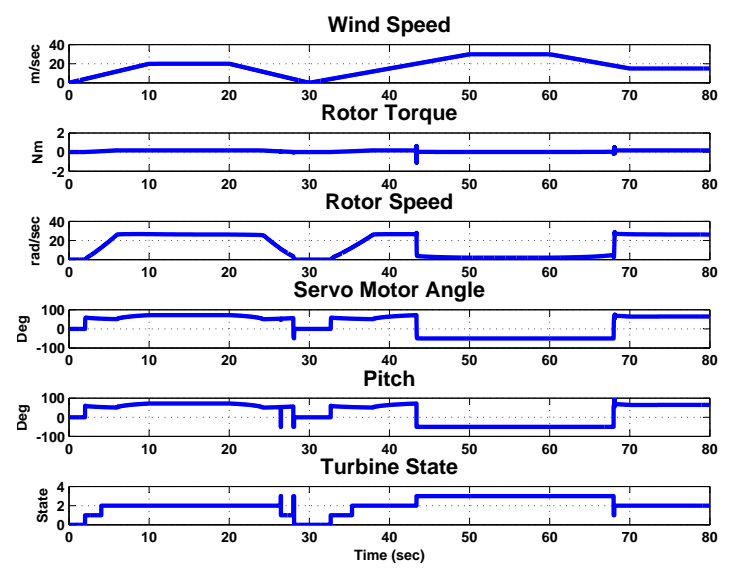

Fig. 9. Simulation Results

designed limit. The simulation results are presented in Figure 9.

The simulation time is step-wise incremented from 0 up to $80 \mathrm{sec}$., with a fixed sample time equal to $1 \mathrm{msec}$. For the simulation, a specific wind speed profile has been created. According to this, the system is simulated for normal operating limits, i.e., 5 - $20 \mathrm{~m} / \mathrm{sec}$ up to $30 \mathrm{sec}$, then up to $30 \mathrm{~m} / \mathrm{sec}$ above $43 \mathrm{sec}$. The simulation results are analyzed w.r.t the turbine control states representing the operational modes (i.e. 0:park, 1:start, 2:generate, 3:brake).

While the simulation provides rich data representing the computation and control of the WTS w.r.t complex environment behavior, system properties however can not be established without analyzing the data. In the next subsection, we present a verification technique to 'exhaustively' analyze the simulation data, w.r.t the overall system timing and causality behavior, towards establishing the system properties. Below, we describe some verification results for the WTS system.

\subsection{Verification}

For WTS, a formal modeling of the corresponding plant and the environment parameters is not possible. Hence, we use simulation data and construct partial functions (input to output values) that represent the computations of the plant components, for instance ROTOR. Also, we use simulation values corresponding to the environment parameters e.g. wind-profile of the WTS. In the next section, we will discuss some aspects about the construction of the relevant partial functions.

Verification of functional properties: Verification of functional properties gives insight into the overall system (architectural) design. For instance, in the WTS case, it is useful to verify the following property: "if the wind speed is within the prescribed limits, the

\footnotetext{
${ }^{6}$ http://www.mathworks.se/products/stateflow/
} 
controller eventually moves to Generate mode". The property can be formulated as a liveness property or leads_to property (denoted by $\rightsquigarrow$, implemented as $-->$ in UPPAAL), as below.

$$
(\text { wS }>=5 \quad \& \& \quad \text { ws }<=20)-->\text { state }==2
$$

Verification of safety-critical properties: One of the safety-critical requirements for the WTS is to fulfill the following property: "the wind turbine moves to Park mode, within 30 s from detecting that the wind speed has crossed the upper limit of $20 \mathrm{~m} / \mathrm{sec}$ ". To verify the property (w.r.t to simulation data), we construct an observer automata for the property as shown in Fig.10, compose the observer with the system model, and verify that the corresponding invariant, the Property (2), holds for the composed model. Note that the urgent channel ' $\mathrm{U}$ !' forces the transition from location B to A without any further delay, when the corresponding transition is enabled.

$$
A \square \text { obs.B implies } x<=30
$$

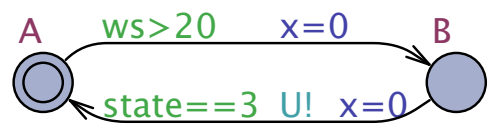

Fig. 10. An observer automata to verify the safety-property: A [ ] obs. B implies $x<=30$

Verifying reachability properties: We can verify reachability of specific control states or computation. For instance, using the Property 3, a reachability property, we can verify that the control state 'Park' (Figure 8.(b)) has been reached (at least once) during the simulation of the WTS. While this may be easily validated using the simulation trace, we can use similar properties to verify specific 'error' states e.g. by extending the behavior model with special 'locations' that are reached if the corresponding 'error' is detected. The presence of these error locations in the simulation data can then be 'exhaustively' verified.

$$
\exists<>\text { MC.Park }
$$

Verifying deadlock-freeness: Using the Property 4, we can verify that the system is deadlock-free, w.r.t overall timed causality behavior of the WTS, as modeled by the corresponding timed automata model. The property is an important validation of the system, which can not be achieved using simulation only, as the corresponding Simulink model does not represent the timing behavior of the system explicitly. Also, the property, when satisfied, verifies the correctness (i.e. consistency) of the timing attributes (Table 1) associated with the system (architectural) design.

$$
A \square \text { (not deadlock) }
$$




\section{Discussion and Lessons-learned}

In this paper, we have presented a formal modeling and verification approach for an industrial system, namely a wind turbine system. The main goal of the work has been to provide formal verification as a complementary analysis method to existing validation techniques based primarily on simulation. We have successfully addressed the following challenges:

- Abstract but expressive system models: Using real-time formalisms such as CCSL and timed automata, we were able to construct intuitive system models amenable for exhaustive verification (w.r.t to timing). With the separation of timing and functional modeling, the technique is scalable to complex system models.

- Verification as complementary analysis to simulation: The verification is based on 'exhaustively' analyzing the simulation data w.r.t the timing behavior of the system. While verification models are expressive in terms of system structure and precise timing behavior, simulation models are suitable to specify plant and the environment, e.g. 'wind profile' modeling in the case of WTS simulation. Thus, the verification approach provides an enhanced simulation-based validation.

The formal modeling approach for the wind turbine system considers the corresponding simulation results to model a suitable abstraction of the plant model. It facilitates constructing a formal model of the WTS, including the plant behavior. This was primarily one of the obstacles in earlier efforts to achieve formal verification of the system. Besides, the formal models were only possible due to expressiveness of real-time semantic formalisms such as CCSL and modeling flexibility in timed automata, as demonstrated in this paper. Further, we believe that the modeling approach is scalable to large complex systems, due to parallel composition of semantic models (timed automata) representing system components. For the verification results, we have considered only the control properties of the system with respect to the overall timed causality behavior. However, we can also consider the data values in the verification, due to timed automata variables. Thus, we have combined the expressiveness of CCSL with modeling capabilities of timed automata.

However, some limitations of our approach do exist. The exhaustiveness of the verification is limited to partial functions constructed using specific instance(s) of simulation. Hence, the approach may be similar to testing-based analysis (albeit model-based). Hence, we need strategies, e.g. choosing suitable simulation step and data profiles, to generate simulation data w.r.t the system properties to be verified. Further, it may be noted that the simulation-extended verification approach presented above may be suitable for data-intensive control systems (e.g. hybrid systems), such as the wind turbine system case study presented in the paper. On the other hand, control-intensive systems may be exhaustively modeled and verifiable using model-checking independent of simulation. 


\section{Conclusion}

In this paper, we have presented a formal modeling and verification approach for an industrial case-study, namely an example wind turbine system. The architectural and behavioral modeling, partially based on the existing system artifacts such as Simulinkmodels, additionally captures precise timing behavior of the system. The modeling approach, based on the real-time formalisms such as CCSL and timed automata, also integrates simulation data to model plant and environment behavior. Based on this, the proposed verification technique using model-checking, enhances the simulation-based system validation. Besides verifying functional properties that validate correctness of the system design, safety-critical properties w.r.t the overall system timing behavior can also be verified. This is clearly an important analysis step forward within existing validation approaches for industrial applications. Thus the paper addresses V\&V challenges in the industrial context, by combining both simulation and verification techniques, paving the way towards scalable application of model-checking for an enhanced validation process. As future work, we intend to investigate requirement-driven strategies to derive the simulation criteria for generating relevant partial functions. This leads to enhanced validation process that can verify useful classes of system properties.

\section{Acknowledgment}

This work was partially funded by Swedish Research Council (project ARROWS), Mälardalen University (Sweden), and ARTEMISIA project iFEST.

\section{References}

1. André, C., Mallet, F., de Simone, R.: Modeling Time(s). In: Models'07. LNCS, vol. 4735, pp. 559-573. Springer (2007)

2. ATESST (Advancing Traffic Efficiency through Software Technology): East-ADL2 specification (March 2008), http://www.atesst.org, 2008-03-20

3. Bouyssounouse, B., Sifakis, J.: Embedded Systems Design: The ARTIST Roadmap for Research and Development (Lecture Notes in Computer Science). Springer-Verlag New York, Inc., Secaucus, NJ, USA (2005)

4. Enoiu, E.P., Marinescu, R., Seceleanu, C., Pettersson, P.: Vital : A verification tool for eastadl models using uppaal port. In: ICECCS'12 (July 2012)

5. Goknil, A., Suryadevara, J., Peraldi-Frati, M.A., Mallet, F.: Analysis Support for TADL2 Timing Constraints on EAST-ADL Models. In: ECSA 2013 : 7th European Conference on Software Architecture. p. 10 pages. LNCS, Montpellier, France (Jul 2013)

6. Larsen, K.G., Pettersson, P., Yi, W.: UPPAAL in a Nutshell. Int. Journal on Software Tools for Technology Transfer 1(1-2), 134-152 (Oct 1997)

7. OMG: UML Profile for MARTE, v1.0. Object Management Group (November 2009), formal/2009-11-02

8. Peraldi-Frati, M.A., Goknil, A., DeAntoni, J., Nordlander, J.: A Timing Model for Specifying Multi Clock Automotive Systems: The Timing Augmented Description Language V2. In: ICECCS 2012. pp. 230-239 (2012) 
9. Slutej, D., Håkansson, J., Suryadevara, J., Seceleanu, C., Pettersson, P.: Analyzing a patternbased model of a real-time turntable system. In: Jens Happe, B.Z. (ed.) 6th International Workshop on Formal Engineering approaches to Software Components and Architectures(FESCA), ETAPS'09, York, UK, March. pp. 161-178. Electronic Notes in Theoretical Computer Science (ENTCS), Vol 253, Elsevier (September 2009)

10. Suryadevara, J., Seceleanu, C., Mallet, F., Pettersson, P.: Verifying MARTE/CCSL mode behaviors using UPPAAL. In: 11th International Conference on Software Engineering and Formal Methods (SEFM 2013) (September 2013)

11. Suryadevara, J.: Validating EAST-ADL timing constraints using UPPAAL. In: 39th Euromicro Conference on Software Engineering and Advanced Applications SEAA 2013) (September 2013) 